\title{
Exome Sequencing Identification of EP300 Mutation in a Proband with Coloboma and Imperforate Anus: Possible Expansion of the Phenotypic Spectrum of Rubinstein-Taybi Syndrome
}

\author{
Koji Masuda ${ }^{a}$ Kazuhiro Akiyama ${ }^{a}$ Michiko Arakawad Eriko Nishib, d \\ Noritaka Kitazawa ${ }^{\text {e }}$ Tsukasa Higuchi ${ }^{f}$ Yuki Katou $^{a}$ Katsuhiko Shirahige ${ }^{a}$ c \\ Kosuke Izumia, d \\ a Institute for Molecular and Cellular Biosciences, Research Center for Epigenetic Disease, The University of \\ Tokyo, Tokyo, b Department of Medical Genetics, Shinshu University Graduate School of Medicine, Matsumoto, \\ 'CREST, Japan Science and Technology Agency, Kawaguchi, and 'Division of Medical Genetics, and Departments of \\ e Ophthalmology and f General Pediatrics, Nagano Children's Hospital, Azumino, Japan
}

\section{Key Words}

Coloboma · EP300 mutation · Imperforate anus ·

Rubinstein-Taybi syndrome

\begin{abstract}
Rubinstein-Taybi syndrome (RSTS) is a multisystem developmental disorder characterized by facial dysmorphisms, broad thumbs and halluces, growth retardation, and intellectual disability. In about $8 \%$ of RSTS cases, mutations are found in EP300. Previously, the EP300 mutation has been shown to cause the highly variable RSTS phenotype. Using exome sequencing, we identified a de novo EP300 frameshift mutation in a proband with coloboma, facial asymmetry and imperforate anus with minimal RSTS features. Previous molecular studies have demonstrated the importance of EP300 in oculogenesis, supporting the possibility that EP300 mutation may cause ocular coloboma. Since a wide
\end{abstract}

phenotypic spectrum is well known in EP300-associated RSTS cases, the atypical phenotype identified in our proband may be an example of rare manifestations of RSTS.

(c) 2015 S. Karger AG, Basel

Rubinstein-Taybi syndrome (RSTS; MIM 180849 and 613684 ) is a multisystem developmental disorder characterized by facial dysmorphisms, broad thumbs and halluces, growth retardation, and intellectual disability [Hennekam, 2006]. In about 55\% of RSTS cases, mutations are found in CREBBP [Hennekam, 2006]. Mutations in EP300 have been identified in up to $8 \%$ of the cases [Roelfsema et al., 2005; Negri et al., 2015]. CREBBP and EP300 both encode histone acetyltransferases; therefore, RSTS is regarded as a genetic syndrome caused by altered epigenetic marks due to the loss of these histone acetyltransferases [Lopez-Atalaya et al., 2012]. Previous-

\section{KARGER 125}

(c) 2015 S. Karger AG, Base

$1661-8769 / 15 / 0062-0099 \$ 39.50 / 0$

E-Mail karger@karger.com

www.karger.com/msy
Kosuke Izumi, MD, PhD

Institute for Molecular and Cellular Biosciences, Research Center for Epigenetic Disease The University of Tokyo

7-3-1 Hongo, Tokyo 113-8654 (Japan)

E-Mail kosukeizumi@iam.u-tokyo.ac.jp 
ly, 15 cases of individuals with EP300 mutations have been reported in the literature. In general, the phenotypic features associated with EP300 mutations are reported to be less severe and quite variable compared to typical RSTS cases with underlying CREBBP mutations [Roelfsema et al., 2005; Bartholdi et al., 2007; Zimmermann et al., 2007; Foley et al., 2009; Bartsch et al., 2010; Tsai et al., 2011; Woods et al., 2014]. However, such phenotypic variation has been identified in cohorts of individuals that are suspected of having RSTS features; therefore, it is possible that EP300 mutations cause phenotypic features that are not considered typical for RSTS.

The recent introduction of exome sequencing has altered the process of defining phenotypic spectra because it has enabled the screening of genetic mutations in an unbiased fashion. Therefore, exome sequencing has expanded the phenotypic spectra of known Mendelian disorders [Izumi et al., 2014]. Here, we report an individual with an EP300 mutation who presented with atypical manifestations for RSTS.

\section{Case Report}

The proband was born to a 33-year-old G1P1 mother after 37 $5 / 7$ weeks of gestation. At approximately 32 weeks of gestation, intrauterine growth retardation was noted. Apgar scores were 8 and 9 at 1 and $5 \mathrm{~min}$, respectively. The birth weight was $1,956 \mathrm{~g}$ $(-1.8 \mathrm{SD})$, length was $42.5 \mathrm{~cm}(-2.5 \mathrm{SD})$, and head circumference was $29.5 \mathrm{~cm}(-1.9 \mathrm{SD})$. After birth, an imperforate anus with an anocutaneous fistula beneath the vaginal opening was diagnosed. The proband was also found to have patent ductus arteriosus (PDA) and developed cardiac failure due to the increased blood flow associated with PDA. The proband underwent PDA ligation at the age of 6 days. Subsequently, because of an imperforate anus, she underwent cutback anoplasty at the age of 22 days. A systemic evaluation revealed bilateral microphthalmia and bilateral iris coloboma as well as optic disc coloboma.

At 14 months of age, her facial dysmorphisms included a prominent forehead, facial asymmetry, bilateral epicanthus, a smaller right palpebral fissure compared to the left side, asymmetric auricle, a broad nasal bridge, and a bulbous nasal tip (fig. 1). There were no hand or feet anomalies, except for slightly broad thumbs and halluces. Her family history was unremarkable. Her development was mildly delayed; she did not begin rolling over and was unable to sit independently at 8 months of age. She started grabbing objects at 3 months. Since the proband had an imperforate anus, she underwent a sacral MRI, but no additional structural abnormalities were detected in the lumbosacral regions. A brain MRI at 9 months did not reveal any structural brain abnormalities such as corpus callosum malformations. There were no inner ear malformations.

A G-band karyotype analysis revealed 46,XX. A fluorescent in situ hybridization analysis using a TUPLE1 probe targeting the 22q11.2 region did not reveal any copy number alterations.
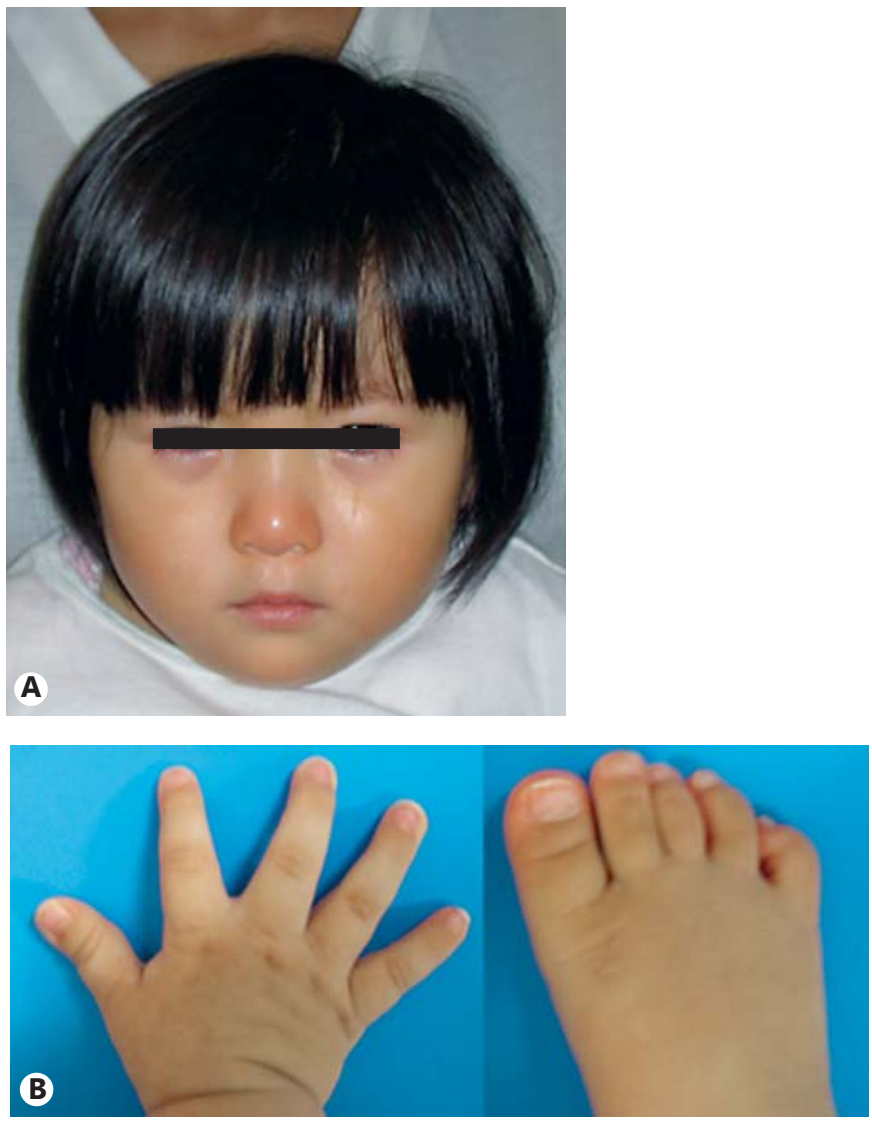

Fig. 1. Physical features of the proband showing facial asymmetry, broad nasal bridge and bulbous nasal tip (A), and slightly broad thumbs and halluces (B).

\section{Materials and Methods}

\section{Exome Sequencing}

Exome sequencing was performed using a protocol approved by the Institutional Review Boards at the University of Tokyo and Nagano Children's Hospital. Genomic DNA samples of the proband and her parents were isolated from peripheral blood using the NucleoSpin Blood Kit (Macherey-Nagel, Düren, Germany). Exome capture was performed with Agilent SureSelect XT Human All Exon V5 (Agilent, Santa Clara, Calif., USA). Using the captured DNA samples, 100-bp paired-end read sequencing was performed by HiSeq 2500 (Illumina, San Diego, Calif., USA).

\section{Bioinformatics Analysis}

The resulting reads were mapped to the human genome hg19 using BWA v0.7.8 with the 'bwa mem-M' option [Li and Durbin, 2009]. We used the Genome Analysis Toolkit (GATK) v3.1.1 [DePristo et al., 2011] and followed 'Best Practices' for SNV and indel discovery and genotyping. After duplicate marking, local realignment around indels, and base quality score recalibration, SNVs and indels for each sample were called independently by HaplotypeCaller. SNVs and indels from each sample were combined by GenotypeGVCFs and then filtered through a variant quality score 


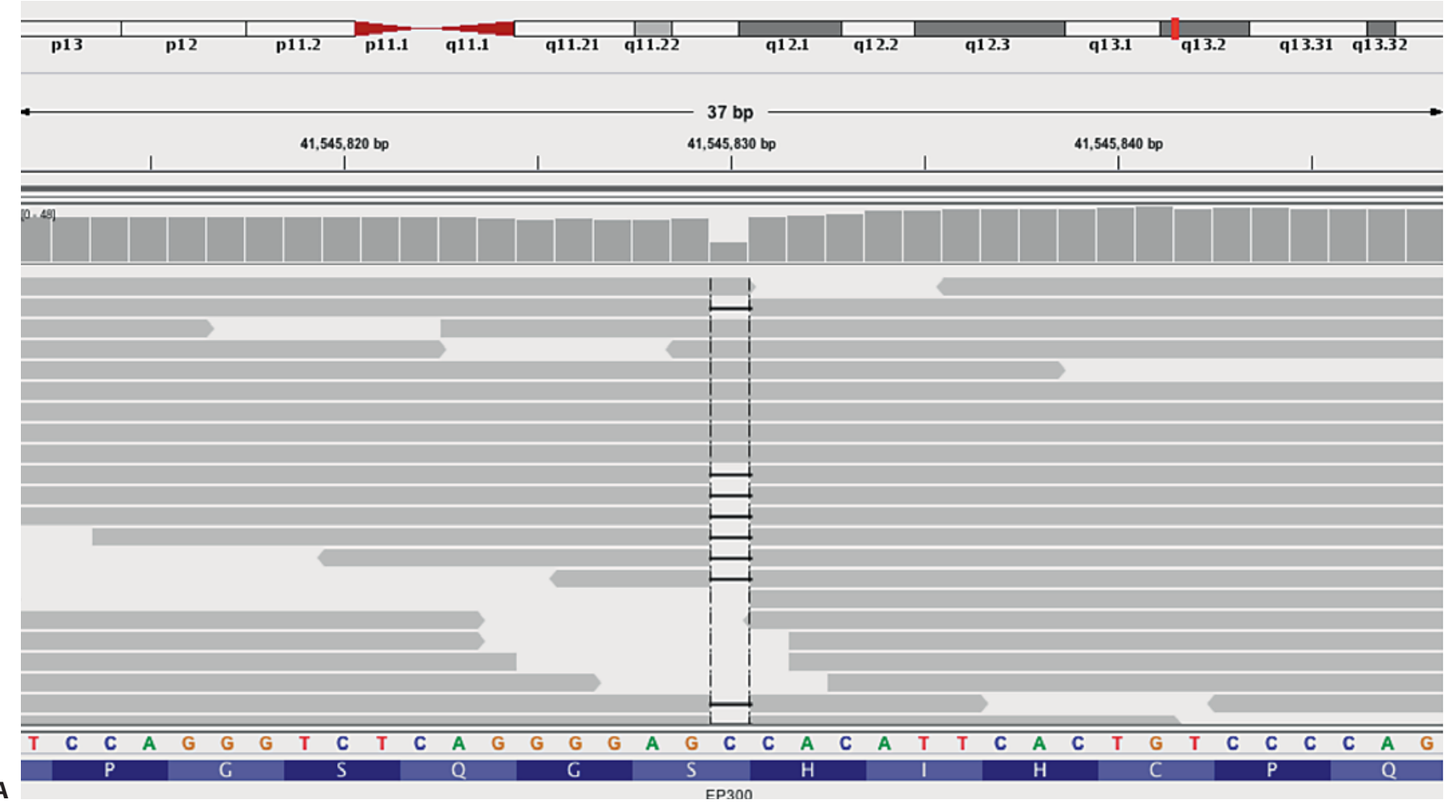

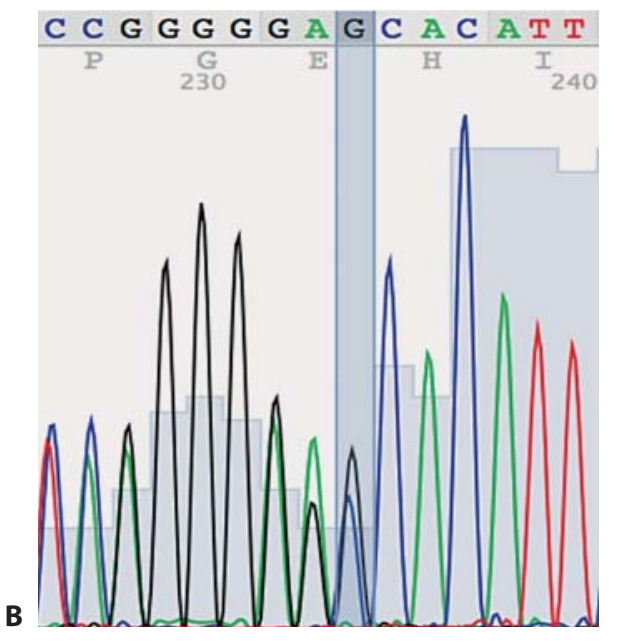

Fig. 2. Identification of the $E P 300$ mutation. A IGV view of the exome sequencing result. EP300 mutation (NM_001429:exon14: c.2445delC:p.S815fs) is demonstrated by the 2 vertical lines. B Sanger sequencing confirmation of the mutation.

recalibration process with default parameters. SNVs and indels with a GQ above 15 were used for further analysis. The effects of variants were predicted by SnpEff v3.6b [Cingolani et al., 2012] and annotated by ANNOVAR [Wang et al., 2010]. Information and statistics regarding sequencing, mapping, coverage, and called variants are summarized in online supplementary table 1 (see www.karger.com/doi/10.1159/000375542).

EP300 Mutation in a Proband with Coloboma

\section{Results}

\section{Exome Sequencing}

After filtering, 38,490, 38,040, and 38,289 variants were confidently called from the father, mother, and proband, respectively. Among 9,197 variants with the presumed critical effect in the annotated genes, 226 de novo variants were identified from the proband. Variants $>5 \times$ coverage were scrutinized. Furthermore, the variants found in only a very small proportion of total reads were eliminated. Only variants in EP300 and KIAA0319 were not reported in SNP database build 129 (http://www. ncbi.nlm.nih.gov/SNP/) and the Human Genetic Variation database (http://www.genome.med.kyoto-u.ac.jp/ $\mathrm{SnpDB} /$ ). Sanger sequencing verified the mutations identified by exome sequencing. The EP300 mutation was a novel frameshift mutation (NM_001429:exon14: c.2445delC:p.H815Tfs*128; fig. 2). This mutation was only found in the proband, and not in either parent, confirming the de novo acquisition of the mutation. The KIAA0319 variant (NM_014809:exon3:c.A127G: p.T43A) was inherited from the asymptomatic father. 


\section{Discussion}

Here, we report a proband with ocular coloboma, facial asymmetry, imperforate anus, and mild developmental delay due to a frameshift mutation in EP300, which was discovered by exome sequencing. Hitherto, 15 individuals with EP300 mutations have been reported in the literature. Although EP300 was initially identified as a second gene underlying RSTS, the characteristic dysmorphic or physical features of RSTS are often mild [Zimmermann et al., 2007]. Therefore, it remains possible that there has been an ascertainment bias against individuals with EP300 mutations. Since our case represents the first proband with minimal RSTS and ocular coloboma in whom a EP300 mutation was identified, it remains unclear whether ocular coloboma is caused by EP300 mutation because there may be other mutations undetected in the proband's genome that could explain her atypical features. Before reaching the conclusion that EP300 mutations cause ocular coloboma, more cases with similar phenotype and EP300 mutations needs to be identified. However, clinical studies characterizing a cohort of RSTS patients and basic research findings support the hypothesis that EP300 mutation may cause ocular coloboma.

Although ophthalmologic manifestations are not regarded as cardinal features of RSTS, the ocular abnormalities are often identified in individuals with RSTS. Frequently observed ocular manifestations are lacrimal duct obstruction, ptosis, strabismus, and refractive errors [van Genderen et al., 2000]. Coloboma is also reported as occasional ocular manifestation of RSTS [van Genderen et al., 2000].

The cardinal function of EP300 in oculogenesis can be inferred from previous studies. In EP300 knockout mice, ocular expression of Pax6 and Sox2 is reduced [Wolf et al., 2013]. Mutations in PAX6 and SOX2 are known etiologies of ocular coloboma in humans, suggesting the importance of proper EP300 expression in ocular embryogenesis [Azuma et al., 2003; Wang et al., 2008]. Furthermore, EP300 was shown to activate PAX2 [Hoffmeister et al., 2002]. PAX2 mutation is associated with renal coloboma syndrome [Sanyanusin et al., 1995]. Therefore, it is reasonable to speculate on the essential role of EP300 in ocular embryogenesis.

Clinically, one of the differential diagnoses was CHARGE syndrome (MIM 214800), based on ocular coloboma, facial asymmetry, and congenital heart defect. CHARGE syndrome is caused by mutations in CHD7 [Vissers et al., 2004]. It is very intriguing to note that a previous chromatin immunoprecipitation-sequencing study revealed genome-wide colocalization between CHD7 and EP300 at gene enhancer elements, suggesting that CHD7 and EP300 may control similar downstream target genes [Schnetz et al., 2010]. In fact, recent articles have revealed a common molecular target of EP300 and CHD7. The molecular interaction between EP300 and p53 is well known, and EP300 protein facilitates the function of p53 [Avantaggiati et al., 1997; Lill et al., 1997]. Recently, p53 was found to be a downstream target of $C H D 7$, and $p 53$ heterozygosity partially rescues the CHARGE syndrome phenotype in Chd7 null mice [Van Nostrand et al., 2014]. Therefore, p53-mediated gene expression alteration may underlay the shared molecular phenomenon explaining the phenotypic similarities between RSTS and CHARGE syndrome.

Previously, the EP300 mutation has been shown to cause the highly variable RSTS phenotype. The recent discovery of an EP300 mutation in a proband with a Cornelia de Lange syndrome-like phenotype using exome sequencing further suggests the pleiotropic effect of EP300 mutations [Woods et al., 2014]. However, the phenotypic spectrum of EP300 mutations has yet to be fully uncovered, and in order to understand its full spectrum, unbiased genetic screening achieved by exome sequencing would be important.

In summary, we report a proband with ocular coloboma, mild intellectual disability, imperforate anus, and minimal RSTS features, in whom an EP300 mutation was identified. Given the basic and clinical research findings, EP300 represents a potential candidate gene for ocular coloboma. Since a wide phenotypic spectrum is well known in EP300-associated RSTS cases, the atypical phenotype identified in our proband may be an example of rare manifestations of RSTS.

\section{Acknowledgement}

The authors greatly appreciate the family members of the proband for permission to publish this case report. The authors thank Mrs. Keiko Nakagawa for technical assistance. This study was funded by Grants-in-Aid for Scientific Research (S) from the Ministry of Education, Culture, Sports, Science and Technology (K.S.).
Masuda/Akiyama/Arakawa/Nishi/ Kitazawa/Higuchi/Katou/Shirahige/Izumi 


\section{References}

Avantaggiati ML, Ogryzko V, Gardner K, Giordano A, Levine AS, Kelly K: Recruitment of p300/CBP in p53-dependent signal pathways. Cell 89:1175-1184 (1997).

-Azuma N, Yamaguchi Y, Handa H, Tadokoro K, Asaka A, et al: Mutations of the PAX6 gene detected in patients with a variety of opticnerve malformations. Am J Hum Genet 72: 1565-1570 (2003).

Bartholdi D, Roelfsema JH, Papadia F, Breuning $\mathrm{MH}$, Niedrist D, et al: Genetic heterogeneity in Rubinstein-Taybi syndrome: delineation of the phenotype of the first patients carrying mutations in EP300. J Med Genet 44:327-333 (2007).

- Bartsch O, Labonté J, Albrecht B, Wieczorek D, Lechno S, et al: Two patients with EP300 mutations and facial dysmorphism different from the classic Rubinstein-Taybi syndrome. Am J Med Genet A 152A:181-184 (2010).

-Cingolani P, Platts A, Wang LL, Coon M, Nguyen $\mathrm{T}$, et al: A program for annotating and predicting the effects of single nucleotide polymorphisms, SnpEff: SNPs in the genome of Drosophila melanogaster strain w1118;iso-2; iso-3. Fly (Austin) 6:80-92 (2012).

-DePristo MA, Banks E, Poplin R, Garimella KV, Maguire JR, et al: A framework for variation discovery and genotyping using next-generation DNA sequencing data. Nat Genet 43: 491-498 (2011).

-Foley P, Bunyan D, Stratton J, Dillon M, Lynch SA: Further case of Rubinstein-Taybi syndrome due to a deletion in EP300. Am J Med Genet A 149A:997-1000 (2009).

Hennekam RCM: Rubinstein-Taybi syndrome. Eur J Hum Genet 14:981-985 (2006).

Hoffmeister A, Ropolo A, Vasseur S, Mallo GV, Bodeker H, et al: The HMG-I/Y-related protein $\mathrm{p} 8$ binds to $\mathrm{p} 300$ and Pax2 trans-activation domain-interacting protein to regulate the trans-activation activity of the Pax $2 \mathrm{~A}$ and
Pax2B transcription factors on the glucagon gene promoter. J Biol Chem 277:2231422319 (2002).

Izumi K, Noon S, Wilkens A, Krantz ID: NKX2.5 mutation identification on exome sequencing in a patient with heterotaxy. Eur J Med Genet 57:558-561 (2014)

Li H, Durbin R: Fast and accurate short read alignment with Burrows-Wheeler transform. Bioinformatics 25:1754-1760 (2009).

Lill NL, Grossman SR, Ginsberg D, DeCaprio J, Livingston DM: Binding and modulation of p53 by p300/CBP coactivators. Nature 387 : 823-827 (1997).

Lopez-Atalaya JP, Gervasini C, Mottadelli F, Spena $S$, Piccione $M$, et al: Histone acetylation deficits in lymphoblastoid cell lines from patients with Rubinstein-Taybi syndrome. J Med Genet 49:66-74 (2012).

-Negri G, Milani D, Colapietro P, Forzano F, Della Monica M, et al: Clinical and molecular characterization of Rubinstein-Taybi syndrome patients carrying distinct novel mutations of the EP300 gene. Clin Genet 87:148-154 (2015).

- Roelfsema JH, White SJ, Ariyürek Y, Bartholdi D, Niedrist D, et al: Genetic heterogeneity in Rubinstein-Taybi syndrome: mutations in both the $C B P$ and EP300 genes cause disease. Am J Hum Genet 76:572-580 (2005).

- Sanyanusin P, Schimmenti LA, McNoe LA, Ward TA, Pierpont ME, et al: Mutation of the PAX2 gene in a family with optic nerve colobomas, renal anomalies and vesicoureteral reflux. Nat Genet 9:358-364 (1995).

Schnetz MP, Handoko L, Akhtar-Zaidi B, Bartels $\mathrm{CF}$, Pereira CF, et al: $\mathrm{CHD} 7$ targets active gene enhancer elements to modulate ES cellspecific gene expression. PLoS Genet 6: e1001023 (2010).

Tsai ACH, Dossett CJ, Walton CS, Cramer AE, Eng PA, et al: Exon deletions of the EP300 and
CREBBP genes in two children with Rubinstein-Taybi syndrome detected by aCGH. Eur J Hum Genet 19:43-49 (2011).

Van Genderen MM, Kinds GF, Riemslag FC, Hennekam RC: Ocular features in Rubinstein-Taybi syndrome: investigation of $24 \mathrm{pa}$ tients and review of the literature. $\mathrm{Br} J \mathrm{Oph}-$ thalmol 84:1177-1184 (2000).

- Van Nostrand JL, Brady CA, Jung H, Fuentes DR, Kozak MM, et al: Inappropriate p53 activation during development induces features of CHARGE syndrome. Nature 514:228-232 (2014).

-Vissers LELM, van Ravenswaaij CMA, Admiraal R, Hurst JA, de Vries BBA, et al: Mutations in a new member of the chromodomain gene family cause CHARGE syndrome. Nat Genet 36:955-957 (2004).

-Wang $\mathrm{K}$, Li M, Hakonarson H: ANNOVAR: functional annotation of genetic variants from high-throughput sequencing data. $\mathrm{Nu}$ cleic Acids Res 38:e164 (2010).

Wang P, Liang X, Yi J, Zhang Q: Novel SOX2 mutation associated with ocular coloboma in a Chinese family. Arch Ophthalmol 126:709713 (2008).

Wolf L, Harrison W, Huang J, Xie Q, Xiao N, et al: Histone posttranslational modifications and cell fate determination: lens induction requires the lysine acetyltransferases CBP and p300. Nucleic Acids Res 41:10199-10214 (2013).

Woods SA, Robinson HB, Kohler LJ, Agamanolis D, Sterbenz G, Khalifa M: Exome sequencing identifies a novel EP300 frame shift mutation in a patient with features that overlap Cornelia de Lange syndrome. Am J Med Genet A 164A:251-258 (2014).

Zimmermann N, Acosta AM, Kohlhase J, Bartsch O: Confirmation of EP300 gene mutations as a rare cause of Rubinstein-Taybi syndrome. Eur J Hum Genet 15:837-842 (2007). 